\title{
Watch-PAT is Useful in the Diagnosis of Sleep Apnea in Patients with Atrial Fibrillation
}

This article was published in the following Dove Press journal:

Nature and Science of Sleep

\author{
Riva Tauman' \\ Murray Berall $\mathbb{D}^{2}$ \\ Richard Berry (D) $^{3}$ \\ Tamar Etzioni ${ }^{4}$ \\ Noam Shrater ${ }^{5}$ \\ Dennis Hwang ${ }^{6}$ \\ Ibrahim Marai $^{7}$ \\ Prasanth Manthena ${ }^{8}$ \\ Anil Rama ${ }^{9}$ \\ Rebecca Spiegel (D) 10 \\ Thomas Penzel (D) ${ }^{11}$ \\ Nira Koren Morag ${ }^{12}$ \\ Giora Pillar $\mathbb{D}^{4}$ \\ 'Sleep Disorders Center, Tel Aviv \\ Souraski Medical Center, Tel Aviv, Israel; \\ ${ }^{2}$ Center of Sleep and Chronobiology, \\ Toronto, ON, Canada; ${ }^{3}$ UF Health Sleep \\ Center, University of Florida, Gainesville, \\ FL, USA; ${ }^{4}$ Technion Faculty of Medicine, \\ Sleep Laboratory, Carmel Medical \\ Center, Haifa, Israel; ${ }^{5}$ Cardiology \\ Department, Soroka Medical Center, \\ Be'er Sheva, Israel; 'Kaiser Permanente \\ Fontana Medical Center, Fontana, CA, \\ USA; ${ }^{7}$ Cardiology Department, Rambam \\ Medical Center, Haifa, Israel; ${ }^{8}$ Sleep \\ Clinic, Kaiser Permanente Los Angeles \\ Medical Center, Los Angeles, CA, USA; \\ ${ }^{9}$ Kaiser Permanente San Jose Medical \\ Center, San Jose, CA, USA; ${ }^{10}$ Stony \\ Brook University Hospital, Stony Brook, \\ NY, USA; "'Charite Universitätsmedizin \\ Berlin, Sleep Medicine Center, Berlin, \\ Germany; ${ }^{12}$ Sackler School of Medicine, \\ Tel Aviv University, Tel Aviv, Israel
}

Correspondence: Riva Tauman Sleep Disorders Center, Tel Aviv Souraski Medical Center, 6 Weitzman Street,

Tel Aviv 6423906, Israel

Tel $+972369746 \mid 4$

Fax + I 97236974634

Email tauman@tlvmc.gov.il
Background: Early diagnosis and treatment of sleep apnea in patients with atrial fibrillation (AF) is critical. The WatchPAT (WP) device was shown to be accurate for the diagnosis of sleep apnea; however, studies using the WatchPAT device have thus far excluded patients with arrhythmias due to the potential effect of arrhythmias on the peripheral arterial tonometry (PAT) amplitude and pulse rate changes.

Purpose: To examine the accuracy of the WP in detecting sleep apnea in patients with AF. Patients and Methods: Patients with AF underwent simultaneous WP and PSG studies in 11 sleep centers. PSG scoring was blinded to the automatically analyzed WP data.

Results: A total of 101 patients with AF (70 males) were recruited. Forty-six had AF episodes during the overnight sleep study. A significant correlation was found between the PSG-derived AHI and the WP- derived AHI $(r=0.80, p<0.0001)$. There was a good agreement between PSG-derived AHI and WP-derived AHI (mean difference of AHI: -0.02 \pm 13.2 ). Using a threshold of $A H I \geq 15$ per hour of sleep, the sensitivity and specificity of the WP were 0.88 and 0.63 , respectively. The overall accuracy in sleep staging between WP and PSG was $62 \%$ with Kappa agreement of 0.42 .

Conclusion: WP can detect sleep apnea events in patients with AF. AF should not be an exclusion criterion for using the device. This finding may be of even greater importance in the era of the COVID19 epidemic, when sleep labs were closed and most studies were home based.

Keywords: home sleep apnea test, WatchPAT, obstructive sleep apnea, atrial fibrillation

\section{Introduction}

Sleep apnea is a common sleep disorder increasingly recognized as a risk for cardiovascular disease. Atrial fibrillation (AF) is the most common cardiac arrhythmia. AF is associated with significant morbidity and mortality. ${ }^{1}$ Sleep apnea is highly prevalent in patients with AF with prevalence estimations ranging from $49 \%$ to $62 \%{ }^{2,3}$ Moreover, several studies have shown that AF recurrence after electrical cardioversion is higher among patients with sleep apnea ${ }^{4-6}$ and sleep apnea treatment leads to $40 \%$ reduction in the risk of $\mathrm{AF}$ recurrence. ${ }^{7,8}$ Sleep apnea is also an independent predictor of stroke in patients with $\mathrm{AF}^{9}$ and reduces the efficacy of antiarrhythmic therapy. ${ }^{10-12}$ Indeed, sleep apnea has been recognized as one of the important modifiable risk factors for AF with the acknowledgement that early diagnosis and treatment of sleep apnea in this population of patients can reduce morbidity and mortality. 8,13

The accepted gold standard for sleep apnea diagnosis is polysomnography (PSG); however, access to PSG is limited. Home Sleep Apnea Testing (HSAT) 
have been therefore developed for the detection of sleep apnea. ${ }^{14}$ The recent COVID19 epidemic further emphasized the importance of the ability to diagnose sleep disordered breathing in the home. Many sleep labs were closed, and most studies were home based. The WatchPAT (peripheral arterial tonometry; WP) technology is a viable alternative to PSG for confirmation of clinically suspected sleep apnea ${ }^{15}$ that is based on peripheral arterial tone signal's amplitude and rate, oxygen saturation and actigraphy. The WP provides accurate sleep/wake and REM/NREM discrimination together with non-REM categorization into deep and light sleep. ${ }^{16-18}$ In addition, the WP provides accurate measurements of snoring and body position. It has recently been shown to be able to distinct obstructive from central sleep apnea. ${ }^{19}$

The WP device has been shown to be accurate for the diagnosis of sleep apnea; however, studies using the WP device have thus far excluded patients with arrhythmias due to the potential effect of arrhythmias on the peripheral arterial tonometry (PAT) amplitude and rate changes. Since arrhythmias in general and AF in particular are quite common in patients with OSA, understanding the accuracy of the WP in detecting sleep apnea in these patients is of substantial importance. Thus, in the present study, we aimed to examine the utility of the WP in detecting sleep apnea in patients with AF.

\section{Patients and Methods}

This was a multicenter study. Participants were recruited as part of a larger study at 11 Medical Centers (US, Canada, Germany and Israel). Full in-lab PSG and WP200U (Itamar Medical, Caesarea, Israel) were conducted simultaneously. External experienced PSG technologist who was blinded to the automatic scoring analysis of the WP manually scored the PSG. The WP signals were analyzed using its automatic software (for more details about the WP technology and automatic analysis see references: 16, 17, 21, 22, 24, 28, 29). Comparisons between the manual PSG scoring results and the WP automatic scoring analysis were conducted. We confirm that all sites obtained ethical approvals and that the study complied with the Declaration of Helsinki. The list of sites:

1. Souraski Medical Center, Tel Aviv, Israel

2. Center of Sleep and Chronobiology, Toronto, ON, Canada

3. UF Health Sleep Center, University of Florida, Gainesville, FL, USA
4. Carmel Medical Center, Haifa, Israel

5. Soroka Medical Center, Be'er Sheva, Israel

6. Kaiser Permanente Fontana Medical Center, Fontana, CA, USA

7. Kaiser Permanente San Jose Medical Center, San Jose, CA, USA

8. Kaiser Permanente Los Angeles Medical Center, Los Angeles, CA, USA

9. Rambam Medical Center, Haifa, Israel

10. Stony Brook University Hospital, Stony Brook, NY, USA

11. Charite Universitätsmedizin Berlin, Sleep Medicine Center, Berlin, Germany.

Informed consent was obtained from all participants.

The inclusion criteria for this subgroup included: 1 . Patients who were previously diagnosed with AF (permanent, persistent or paroxysmal) and suspected to have sleep apnea, 2. Age between 17 and 90,3. Subject able to read, understand and sign the informed consent, 4. Willing to sleep with the WP device and the PSG simultaneously in the sleep lab.

Exclusion criteria included: finger deformity that precluded adequate sensor appliance, use of short acting nitrates for less than 3 hours before the study and use of alpha blockers.

\section{Polysomnography}

In the present study, the reference used for comparison was in-lab PSG. In-lab PSG was FDA-approved from multiple manufacturers used in the eleven study sites, all according to the site regular clinical practice and compliant with accepted standards. Manual scoring was conducted according to the American Academy of Sleep Medicine guidelines. ${ }^{20}$ Moderate-severe sleep apnea was defined as AHI $\geq 15$ /hour.

\section{WatchPAT System}

The WP system is a home testing (HSAT) system that is grounded on a wrist-worn device with a finger probe that obtains Peripheral Arterial Tonometry (PAT) signals and oxygen saturation levels, a snoring and body position sensor that is positioned under the sternal notch and accelerometer that is embedded in the wrist unit. The WP algorithm detects respiratory (apnea/hypopnea) events, sleep/wake status, and determines sleep stages.

As previously described, ${ }^{21,22}$ the WP finger probe measures changes in vascular tone at the fingertip. These changes are controlled by $\alpha$-adrenergic receptors of 


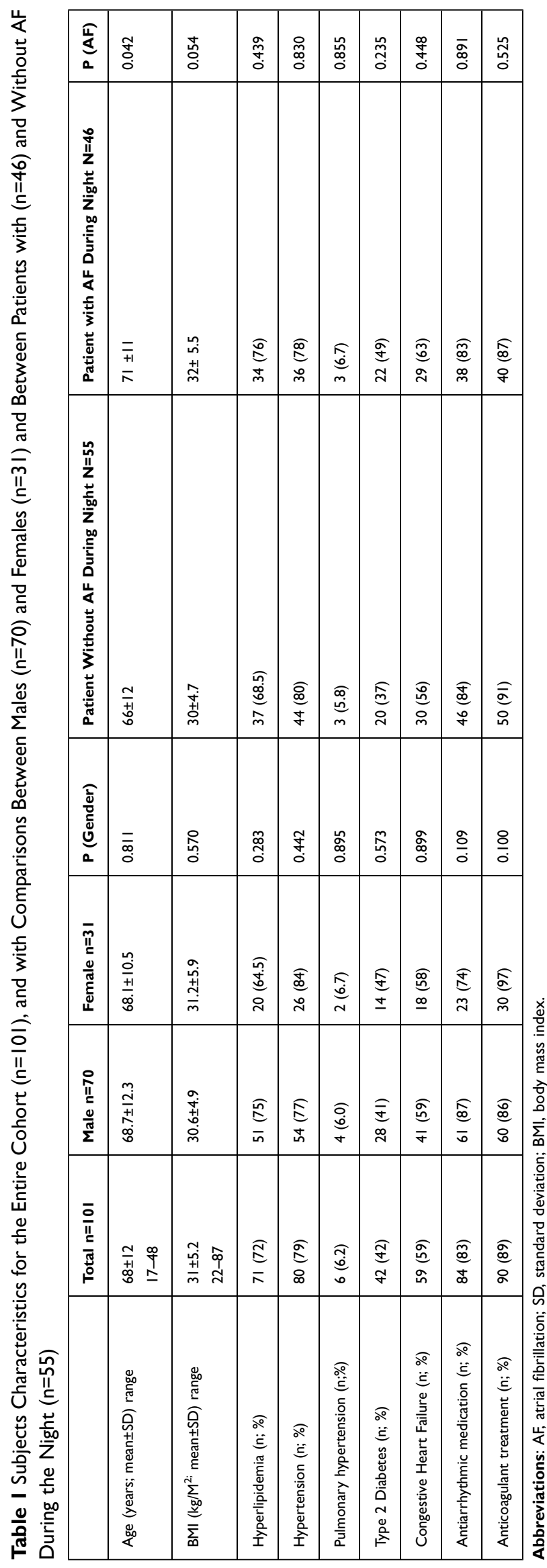

vascular smooth muscle of the finger vascular bed and thus reflect sympathetic nervous system activity of the patient. ${ }^{23}$ The WP measures these changes in the vascular tone at the fingertip, with transient vasoconstriction events and increase pulse rate associated with sympathetic activation that typically terminates respiratory events. ${ }^{21}$

The autonomic basis for the transient events of vasoconstriction provides a sensitive and accurate method for detecting arousals, that are noticeably more sensitive than electroencephalographically detected cortical arousals. These autonomic subcortical arousals are more sensitive markers of arousal than EEG, even in children. ${ }^{24}$ Subcortical arousals are of particular importance in adults, in whom significant percentage of respiratory events are not associated with cortical arousals but rather related to subcortical autonomic arousals. Indeed, $10 \%-25 \%$ of actual apneic events were not related to cortical arousals. ${ }^{25}$

\section{Statistical Analysis}

Descriptive statistics are presented as mean (SD) or count (\%) as applicable. The AHI obtained from the WP device and from PSG were compared as descriptive statistics and compared using Pearson correlation and Bland-Altman. Sensitivity and specificity of the WP in diagnosis moderate-severe sleep apnea were compared to the PSG, ROC and AUC were used. Agreement of sleep stages measured by WP vs PSG was computed using overall agreement and kappa level of agreement.

\section{Results}

A total of 101 patients with AF (70 males) were included in the analysis. Of those, 12 had permanent AF, 15 had persistent AF, 46 had paroxysmal AF and 28 had unknown type recorded on their medical history. Subject's characteristics of the entire cohort and comparisons between males and females are presented in Table 1. There were no significant differences between males and females. Of the 101 patients recruited, 46 had AF episodes during the overnight sleep study, as detected from an ECG channel recorded in the PSG and analyzed by certified ECG interpreter. Of those, $38(83 \%)$ had AF throughout the entire night and $8(17 \%)$ had AF only part of the night. The presence of AF did not cause significant non-valid PAT signal. The mean excluded sleep time was $4.4 \%$ in the 46 patients with active AF compared with $6.4 \%$ in patients without active AF.

The mean AHI derived from the PSG of the entire cohort was $34.0 \pm 20.3$ (range: 0.9-90.1) and the mean AHI derived 


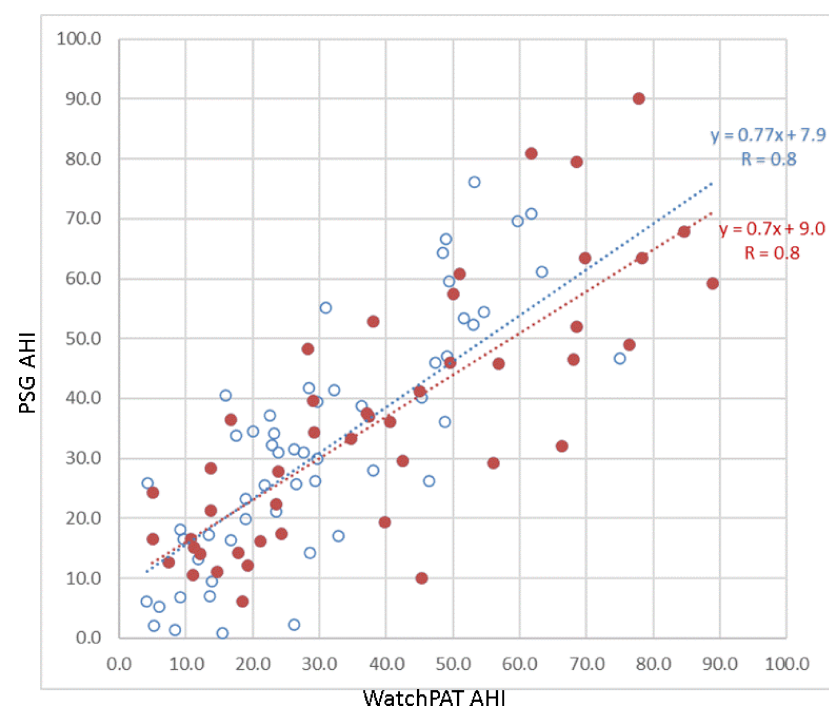

Figure I Correlation between AHI assessed by PSG and by the WP for the entire cohort of $\mathrm{IOI}$ subjects with AF (red dots: patients with AF during the night, blue dots: patients without AF during the night).

from the WP device was $34.1 \pm 21.2$ (range: 4.1-88.9). As shown in Figure 1, a significant correlation was found between the PSG-derived AHI and the WP-derived AHI $(\mathrm{r}=0.80, \mathrm{p}<0.0001)$. This correlation remained significant for the 46 subjects experiencing $\mathrm{AF}$ episodes during the sleep study $(\mathrm{r}=0.8, \mathrm{p}<0.01)$ and for the 38 subjects experiencing AF throughout the entire night $(\mathrm{r}=0.82, \mathrm{p}<0.01)$. This correlation was also significant for the 84 subjects using antiarrhythmic medications $(\mathrm{r}=0.82, \mathrm{p}<0.01)$.

Figure 2 shows the Bland-Altman plot used to assess the agreement between PSG-derived AHI and WP-derived AHI. Mean difference of AHI between the two systems was $-0.02 \pm 13.2$. Using paired test analysis, no difference was found between PSG-derived AHI and WP-derived

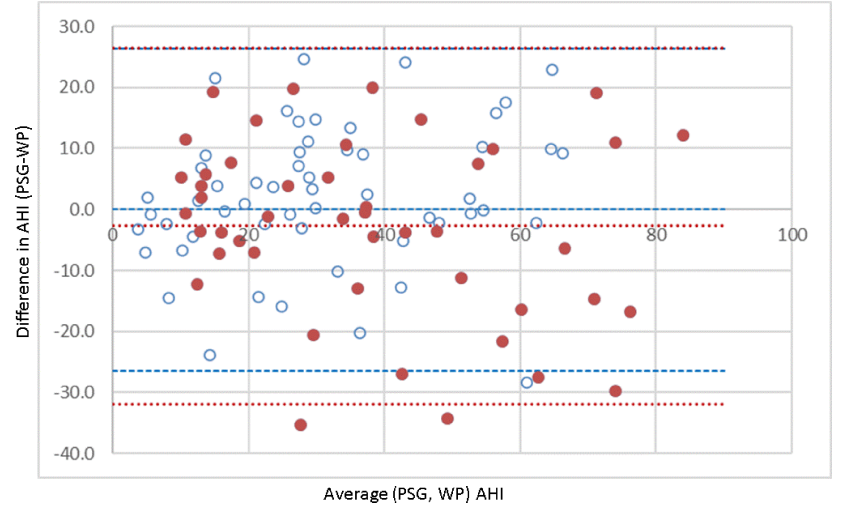

Figure 2 The Bland-Altman plot assessing the agreement between PSG-derived $\mathrm{AHI}$ and WP-derived $\mathrm{AHI}$ for the entire cohort (red dots: patients with $\mathrm{AF}$ during the night, blue dots: patients without AF during the night).

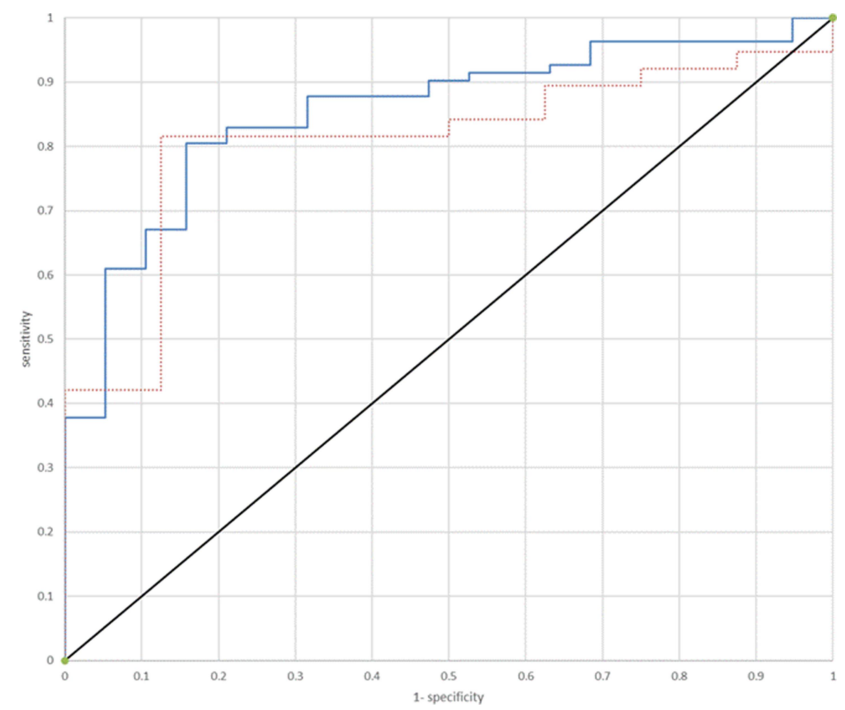

Figure 3 ROC curve for WP-derived $A H I$ to identify patients with $A H I \geq 15$ per hour of sleep (red dots: patients with AF during the night, blue dots: patients without AF during the night).

AHI ( $\mathrm{p}=0.99)$. Subgroup analysis, including only subjects with active AF episodes or only subjects using antiarrhythmic medications, also showed no significant difference between the AHI of the 2 systems $(\mathrm{p}=0.21$ and $\mathrm{p}=0.998$, respectively).

Using a threshold of $\mathrm{AHI} \geq 15$ per hour of sleep, the sensitivity and specificity of the WP for all 101 subjects were 0.88 and 0.63 , respectively, with PPV of 0.91 and NPV of 0.55. For the 46 subjects that had AF during the night, the sensitivity and specificity, were 0.84 and 0.5 , respectively (PPV of 0.89 and NPV of 0.4). Figure 3 shows the ROC curve for WP-derived AHI to identify patients with AHI $\geq 15$ per hour of sleep for both the entire cohort and only for patients who had AF during the night. The area under the curve was 0.85 for the entire group and 0.81 for the patients with AF.

The overall accuracy in sleep staging between WP and PSG based on epoch-by-epoch comparison was $62 \%$ with Kappa agreement of 0.42 . Of note, the accuracy in sleep staging in patients without $\mathrm{AF}$ episodes during the night was $63.4 \%$ with Kappa agreement of 0.45 .

\section{Discussion}

The rising burden of AF has raised the need for strategies aiming to lower the risk of AF development and progression. Sleep apnea has been recognized as one of the important modifiable risk factors for $\mathrm{AF}$ with the recognition that early diagnosis and treatment of sleep apnea in this population of patients is critical. ${ }^{13}$ Early diagnosis of 
sleep apnea in this population can lead to early intervention with reduction of morbidity and mortality. Indeed, international professional societies recommend screening patients with AF for sleep apnea. ${ }^{26,27}$

The findings of the present study indicate that the WP technology which is an HSAT can detect sleep apnea events in patients with AF with and without nocturnal active AF episodes with accuracy similar to its accuracy in the general population. ${ }^{15}$ These findings suggest that the WP, a simple-to apply and minimally disruptive ambulatory sleep testing device, can be used for diagnosis when sleep apnea is suspected in patients with AF.

In the general population, HSAT has gained popularity due to the limited availability of the gold standard in lab PSG. Since the WP wrist-worn device was introduced in the early 2000s, there has been much clinical research to assess the accuracy and feasibility of the technology for identifying sleep apnea. ${ }^{21,22,28-30}$ The WP technology has been shown to be an accurate alternative to PSG with high correlation and good agreement of AHI with attended in lab PSG. ${ }^{28-31}$ The present multicenter study indicates that the WP technology can be used for the diagnosis of sleep apnea in patients with AF.

In previous WP validation studies, patients with arrhythmias were excluded due to a concern regarding its accuracy in the presence of non-sinus rhythms. This concern was originated from the WP algorithm detecting respiratory events based on the combination of peripheral/digital arterial vasoconstriction, oxygen saturation and pulse rate. The findings of the present study indicate that this concern was not justified and that the WP technology is able to detect sleep apnea events and to provide reasonably analyses of sleep stages in AF patients similar to the general population. Moreover, we show that the presence of AF events during the night or the use of antiarrhythmic drugs did not decrease the precision of this technology. In fact, the presence of AF episodes throughout the night had no significant effect on the PAT signal or the time used in the analysis $(4.4 \%$ in the 46 patients with active AF compared with $6.4 \%$ in patients without active AF). Therefore, a diagnosis of AF, the use of antiarrhythmic medications and even active AF episodes throughout the night should not be an exclusion criterion for using the WP device. Nevertheless, as in any ambulatory testing, irrespective of the presence of AF, cases of high clinical suspicious for OSA with normal HSAT results should be referred to a formal in lab polysomnography.
In the present study, we assessed the agreement between WP and PSG according to the AASM scoring guidelines (using the recommended scoring of hypopnea: 3\% oxygen desaturation or the event is associated with an arousal). Using a threshold of $\mathrm{AHI} \geq 15$ per hour of sleep, the sensitivity and specificity of the WP for the entire cohort of patients with AF were 0.88 and 0.63 , respectively, and the area under the curve of the ROC curve for identifying AHI $\geq 15$ was 0.85 . Similarly, the overall agreement in sleep staging between WP and PSG in our cohort was $62 \%$ with Kappa agreement of 0.42 , which is very close to the accuracy found in the general population. ${ }^{16}$

Effective and accurate diagnostic tools that are easy to use with less dependence on skilled technologists could increase the availability of testing in patients with AF and the willingness of patients to undergo sleep study as patients prefer home-sleep studies to attended studies. ${ }^{32}$ Since the identification of sleep apnea in patients with AF is clinically crucial and treatment of sleep apnea can reduce morbidity and mortality, the addition of the WP technology as an alternative sleep testing at home may improve the management of these patients. As AF and sleep apnea are both chronic conditions, our findings showing the ability of the WP to detect sleep apnea is these patients irrespective of the use of anti-arrhythmic therapy may facilitate the overall management of these patients. The fact that the WP is a simple-to-apply and minimally disruptive ambulatory device makes it also suitable for repeated sleep studies throughout the course of disease if clinically indicated.

The limitations of the current study are the inclusion of patients with AF only and no other arrhythmias. Therefore, the results of the present study cannot be generalized for other arrhythmias. Another limitation is the fact that out of the 101 participants diagnosed with AF, only 46 subjects had AF episodes during the overnight sleep study. However, we think that our cohort of patients with the diagnosis of AF reflects well the population of patients with $\mathrm{AF}$ in which the majority of patients have paroxysmal events. Another limitation is the imbalance of subjects under and above 15 events/hour. In addition, the current study focused on OSA diagnosis in the presence of AF, and not on sleep times or stages which were previously published. ${ }^{16,17}$ Potentially, AF could have changed the accuracy of WP-based sleep time and stages scoring. However, we believe that the scored sleep times by PSG and WP are already taken into consideration as the denominator for the SDB indices calculated. Finally, the 
WP was not tested in the home environment where it is intended to be used.

\section{Conclusion}

The findings of the present study suggest that WP can successfully detect sleep apnea events in patients with $\mathrm{AF}$ and that AF should not be an exclusion criterion for using the device.

\section{Disclosure}

Thomas Penzel reports grants from Itamar, Cidelec, and Resmed and grants and personal fees from Philips, and Löwenstein Medical, during the conduct of the study; and is a shareholder of Advanced Sleep Research, Somnico, and The Siestagroup, outside the submitted work. Giora Pillar reports grants from Itamar Medical, during the conduct of the study. The authors report no other potential conflicts of interest in this work.

\section{References}

1. Miyasaka Y, Barnes ME, Gersh BJ, et al. Secular trends in incidence of atrial fibrillation in Olmsted County, Minnesota, 1980 to 2000, and implications on the projections for future prevalence. Circulation. 2006;114(2):119-125. doi:10.1161/CIRCULATIONAHA.105.595140

2. Gami AS, Pressman G, Caples SM, et al. Association of atrial fibrillation and obstructive sleep apnea. Circulation. 2004;110 (4):364-367. doi:10.1161/01.CIR.0000136587.68725.8E

3. Stevenson IH, Teichtahl H, Cunnington D, Ciavarella S, Gordon I, Kalman JM. Prevalence of sleep disordered breathing in paroxysmal and persistent atrial fibrillation patients with normal left ventricular function. Eur Heart J. 2008;29(13):1662-1669. doi:10.1093/eurheartj/ehn214

4. Mazza A, Bendini MG, Cristofori M, et al. Baseline apnoea/hypopnoea index and high-sensitivity C-reactive protein for the risk of recurrence of atrial fibrillation after successful electrical cardioversion: a predictive model based upon the multiple effects of significant variables. Europace. 2009;11(7):902-909. doi:10.1093/europace/ eup107

5. Linz D, Hohl M, Ukena C, et al. Obstructive respiratory events and premature atrial contractions after cardioversion. Eur Respir J. 2015;45(5):1332-1340. doi:10.1183/09031936.00175714

6. Linz D, McEvoy RD, Cowie MR, et al. Associations of obstructive sleep apnea with atrial fibrillation and continuous positive airway pressure treatment: a review. JAMA Cardiol. 2018;3(6):532-540.

7. Qureshi WT, Nasir UB, Alqalyoobi S, et al. Meta-analysis of continuous positive airway pressure as a therapy of atrial fibrillation in obstructive sleep apnea. Am J Cardiol. 2015;116(11):1767-1773. doi:10.1016/j.amjcard.2015.08.046

8. Shukla A, Aizer A, Holmes D, et al. Effect of obstructive sleep apnea treatment on atrial fibrillation recurrence: a meta-analysis. JACC Clin Electrophysiol. 2015;1(1-2):41-51. doi:10.1016/j.jacep.2015.02.014

9. Yaranov DM, Smyrlis A, Usatii N, et al. Effect of obstructive sleep apnea on frequency of stroke in patients with atrial fibrillation. $\mathrm{Am}$ J Cardiol. 2015;115(4):461-465. doi:10.1016/j.amjcard.2014.11.027

10. Szymanski FM, Filipiak KJ, Platek AE, et al. Presence and severity of obstructive sleep apnea and remote outcomes of atrial fibrillation ablations - a long-term prospective, cross-sectional cohort study. Sleep Breath. 2015;19(3):849-856.
11. Neilan TG, Farhad H, Dodson JA, et al. Effect of sleep apnea and continuous positive airway pressure on cardiac structure and recurrence of atrial fibrillation. $J$ Am Heart Assoc. 2013;2(6):e000421. doi:10.1161/JAHA.113.000421

12. Monahan K, Brewster J, Wang L, et al. Relation of the severity of obstructive sleep apnea in response to anti-arrhythmic drugs in patients with atrial fibrillation or atrial flutter. Am J Cardiol. 2012;110(3):369-372. doi:10.1016/j.amjcard.2012.03.037

13. Lau DH, Nattel S, Kalman JM, Sanders P. Modifiable risk factors and atrial fibrillation. Circulation. 2017;136(6):583-596. doi:10.1161/ CIRCULATIONAHA.116.023163

14. Krishnaswamy U, Aneja A, Kumar RM, Kumar TP. Utility of portable monitoring in the diagnosis of obstructive sleep apnea. $J$ Postgrad Med. 2015;61(4):223-229. doi:10.4103/00223859.166509

15. Yalamanchali S, Farajian V, Hamilton C, Pott TR, Samuelson CG, Friedman M. Diagnosis of obstructive sleep apnea by peripheral arterial tonometry: meta-analysis. JAMA Otolaryngol Head Neck Surg. 2013;139(12):1343-1350. doi:10.1001/jamaoto.2013.5338

16. Hedner J, White DP, Malhotra A, et al. Sleep staging based on autonomic signals: a multi-center validation study. J Clin Sleep Med. 2011;7(3):301-306. doi:10.5664/JCSM.1078

17. Herscovici S, Pe'er A, Papyan S, Lavie P. Detecting REM sleep from the finger: an automatic REM sleep algorithm based on peripheral arterial tone (PAT) and actigraphy. Physiol Meas. 2007;28 (2):129-140. doi:10.1088/0967-3334/28/2/002

18. Lavie P, Schnall RP, Sheffy J, Shlitner A. Peripheral vasoconstriction during REM sleep detected by a new plethysmographic method. Nat Med. 2000;6(6):606. doi:10.1038/76135

19. Pillar G, Berall M, Berry R, et al. Detecting central sleep apnea in adult patients using watchPAT-a multicenter validation study. Sleep Breath. 2020;24(1):387-398. doi:10.1007/s11325-019-01904-5

20. Berry RB, Brooks R, Gamaldo C, et al. AASM scoring manual updates for 2017 (Version 2.4). J Clin Sleep Med. 2017;13 (5):665-666. doi:10.5664/jcsm.6576

21. Schnall RP, Shlitner A, Sheffy J, Kedar R, Lavie P. Periodic, profound peripheral vasoconstriction-a new marker of obstructive sleep apnea. Sleep. 1999;22(7):939-946.

22. Bar A, Pillar G, Dvir I, Sheffy J, Schnall RP, Lavie P. Evaluation of a portable device based on peripheral arterial tone for unattended home sleep studies. Chest. 2003;123(3):695-703. doi:10.1378/ chest.123.3.695

23. Pillar G, Bar A, Betito M, et al. An automatic ambulatory device for detection of AASM defined arousals from sleep: the WP100. Sleep Med. 2003;4(3):207-212. doi:10.1016/S1389-9457(02)00254-X

24. Tauman R, O’Brien LM, Mast BT, Holbrook CR, Gozal D. Peripheral arterial tonometry events and electroencephalographic arousals in children. Sleep. 2004;27(3):502-506. doi:10.1093/sleep/27.3.502

25. Eckert DJ, Younes MK. Arousal from sleep: implications for obstructive sleep apnea pathogenesis and treatment. J Appl Physiol. 2014;116(3):302-313. doi:10.1152/japplphysiol.00649.2013

26. Kirchhof P, Benussi S, Kotecha D, et al. 2016 ESC guidelines for the management of atrial fibrillation developed in collaboration with EACTS. Eur Heart J. 2016;37(38):2893-2962.

27. Calkins H, Hindricks G, Cappato R, et al. 2017 HRS/EHRA/ECAS/ APHRS/SOLAECE expert consensus statement on catheter and surgical ablation of atrial fibrillation. Europace. 2018;20(1):e1-e160.

28. Choi JH, Kim EJ, Kim YS, et al. Validation study of portable device for the diagnosis of obstructive sleep apnea according to the new AASM scoring criteria: watch-PAT 100. Acta Otolaryngol. 2010;130 (7):838-843. doi:10.3109/00016480903431139

29. Pittman SD, Ayas NT, MacDonald MM, Malhotra A, Fogel RB, White DP. Using a wrist-worn device based on peripheral arterial tonometry to diagnose obstructive sleep apnea: in-laboratory and ambulatory validation. Sleep. 2004;27(5):923-933. doi:10.1093/ sleep/27.5.923 
30. Zou D, Grote L, Peker Y, Lindblad U, Hedner J. Validation a portable monitoring device for sleep apnea diagnosis in a population based cohort using synchronized home polysomnography. Sleep. 2006;29 (3):367-374. doi:10.1093/sleep/29.3.367

31. Choi JH, Lee B, Lee JY, Kim HJ. Validating the watch-PAT for diagnosing obstructive sleep apnea in adolescents. J Clin Sleep Med. 2018;14(10):1741-1747. doi:10.5664/jcsm.7386
32. Kundel V, Shah N. Impact of portable sleep testing. Sleep Med Clin. 2017;12(1):137-147.

\section{Publish your work in this journal}

Nature and Science of Sleep is an international, peer-reviewed, open access journal covering all aspects of sleep science and sleep medicine, including the neurophysiology and functions of sleep, the genetics of sleep, sleep and society, biological rhythms, dreaming, sleep disorders and therapy, and strategies to optimize healthy sleep.

Submit your manuscript here: https://www.dovepress.com/nature-and-science-of-sleep-journa|
The manuscript management system is completely online and includes a very quick and fair peer-review system, which is all easy to use. Visit http://www.dovepress.com/testimonials.php to read real quotes from published authors. 